\title{
Safe Controller Synthesis for Data-Driven Differential Inclusions
}

\author{
Mohamadreza Ahmadi, Arie Israel, and Ufuk Topcu
}

\begin{abstract}
We consider the problem of designing finite-horizon safe controllers for a dynamical system for which no explicit analytical model exists and limited data only along a single trajectory of the system are available. Given samples of the states and inputs of the system, and additional side information in terms of regularity of the evolution of the states, we synthesize a controller such that the evolution of the states avoid some prespecified unsafe set over a given finite horizon. Motivated by recent results on Whitney's extension theorem, we use piecewisepolynomial approximations of the trajectories based on the data along with the regularity side information to formulate a datadriven differential inclusion model that can predict the evolution of the trajectories. For these classes of data-driven differential inclusions, we propose a safety analysis theorem based on barrier certificates. As a corollary of this theorem, we demonstrate that we can design controllers ensuring safety of the solutions to the data-driven differential inclusion over a finite horizon. From a computational standpoint, our results are cast into a set of sumof-squares programs whenever the certificates are parametrized by polynomials of fixed degree and the sets are semi-algebraic.
\end{abstract}

\section{INTRODUCTION}

Learning-based methods have been successful in modeling and controlling many dynamical systems [1], [2]. These methods often require a large number of system runs (e.g., trajectories from different initial conditions) over a long time span, to achieve reasonable performance. However, for a relatively broad class of systems, collecting large sums of data can be too cumbersome or not viable at all. The scarcity of the available data is particularly noticeable for safety-critical systems, in which an abrupt change in system model can result in catastrophic control failures. For instance, it is not practically possible to test and collect data from all possible failure scenarios for an unmanned vehicle [3]. Furthermore, for safety-critical systems, we need to construct a model that can be used to predict the behavior of the system, and the construction of and control with such models should not incur a high computational cost (as opposed to conventional learning methods).

Recent studies have shown that certain mathematical models in the form of differential equations can be extracted from data [4]. In particular, [5] studied the problem of finding system dynamics when the system follows Lagrangian mechanics. Also, see [6] for a method that can extract chaotic polynomial differential equations from noisy data and relies on the ergodicity property of the data such that the central limit theorem can be applied. However, these methods often require large amounts of training data, which may not be

M. Ahmadi is with the California Institute of Technology, 1200 E. California Blvd., MC 104-44, Pasadena, CA 91125, USA. e-mail: ( $\{$ mrahmadi $\} @$ caltech.edu). U. Topcu and Arie Israel are with the University of Texas at Austin, 2515 Speedway Stop C1200 Austin, Texas 78712-1202, USA. e-mail: ( $\{$ arie, utopcu\}@utexas.edu). The work has been supported partly by AFOSR FA9550-19-1-0005 and NSF 1646522. available. In the control literature, system analysis based on input-output data or input-state data is not new. blueSystem identification techniques [7] have studied the problem of finding a model of the system based on data. Recently, online system identification techniques have been used to determine the parameters of models of autonomous ground [8] and underwater [9] vehicles. Nevertheless, the available methods for online system identification are "data-hungry" or computationally expensive, especially if they require a validation stage. Adaptive control techniques [10] also studied controller synthesis methods for systems in which the system model is known up to a parametrization (see the recent adaptive control method for "monitoring" the controllers suggested by the learning algorithm online, and rejecting controllers leading to instability for linear systems [11] and an adaptive stabilization method for large-scale dynamical systems with uncertain, time-varying parameters [12]). Such parametrization of the system dynamics is not often available, for instance, in the case of an abrupt system change.

One fundamental issue for safety critical systems is to ensure the system behaves safely or guarantee that the system avoids certain unsafe behavior. If the system model is given, verifying safety is a familiar subject to the control community [13], [14]. One of the methods for safety verification relies on the construction of a function of the states, called the barrier certificate [14]. Barrier certificates have shown to be useful in several system analysis and control problems blueincluding bounding moment functionals of stochastic systems [15], safety analysis of systems described by partial differential equations [16], and safety verification of refrigeration systems [17]. It was also proved in [18] that for every safe dynamical system (defined in the appropriate sense), there exists a barrier certificate. To the authors' knowledge, the only article that applied barrier certificates for system analysis based on data is [19]. However, the latter method requires large amounts of data, as well.

Apart from safety analysis, several studies considered the so called control barrier functions as a means to render the solutions of a system safe. In [20], the authors, inspired by the notion of control Lyapunov functions [21], introduced control barrier functions. This formulation, however, requires a one-dimensional control signal. In the same vein, [22] demonstrated that one can simultaneously search for a safe and stabilizing controller. Alternatively, [23] proposed control barrier functions with a fixed logarithmic structure as a function of the unsafe set (also see the recent application to bipedal locomotion [24]). Recently, it was demonstrated that control barrier functions can be used in reinforcement learning to ensure safety in continuous control tasks [25]. Yet, this method also requires large amount of data from different initial conditions and "crude" model information. 
In this paper, we study safety analysis and safe control of systems for which limited data in terms of state and input samples from a single trajectory is available (by limited, we imply that the number of data samples is not large enough for determining the complete dynamics using a system identification or machine learning method). Such scenarios arise, in particular, in the case of an abrupt change in system dynamics. For instance, consider an unmanned aerial vehicle flying on a specified trajectory whose wings incur severe damage due to external conditions such as a wind gust. In this case, we require an on-the-fly safe controller that ensures safe landing given the data from only one trajectory. This research is motivated by the recent works on Whitney's extension problem [26] on finding interpolants with optimal regularity constants in terms of the $\mathcal{C}^{n}$-norms. Following the footsteps [26], [27] showed that the cubic spline polynomials are the best interpolants in terms of minimizing the Lipschitz constant $\left(\mathcal{C}^{0}\right.$-norm) for twice continuously differentiable trajectories and proposed computational methods on how to find these interpolants. Accordingly, we build a data-driven differential inclusion model that can be used to predict the evolution of system trajectories based on the piecewise-polynomial (cubic spline) approximation of the state and input data and some regularity information on the evolution of system state. Equipped with this data-driven model in terms of convex differential inclusions, we formulate a safety analysis theorem based on barrier certificates for differential inclusions using notions from set-valued analysis [28] and the theory of differential inclusions [29]. This barrier certificate is a possibly non-smooth function of the states and time satisfying two inequalities along the solutions of the datadriven differential inclusion. Then, we present conditions to synthesize controllers ensuring safety of the latter differential inclusions, without imposing any a priori fixed structure on the barrier functions. We evince that both the analysis and controller synthesis methods can be cast into a set of sum-ofsquares programs whenever the certificates are parametrized by polynomials of fixed degree and the sets are semi-algebraic.

Preliminary results on this work were discussed in [30], in which we brought forward the safety analysis method based on barrier certificates for the data-driven models. In the current paper, we propose a controller synthesis algorithm for safety of the data-driven models and a computational method based on sum-of-squares optimization to synthesize the safe controllers.

The paper is organized as follows. The next section presents the notation and some preliminary mathematical definitions. In Section III, we show how the data-driven differential inclusion models are constructed from the piece-wise polynomial approximation of the data. In Section IV, we propose a method based on barrier certificates for safety analysis of differential inclusions and a method for designing safe controllers for systems with limited data. Section V describes a computational approach for finding barrier certificates and designing safe controllers based on polynomial optimization. In Section VI, we illustrate the proposed method by an example. Finally, Section VII concludes the paper and provides directions for future research.

Notation: The notations employed in this paper are relatively straightforward. $\mathbb{R}_{\geq 0}$ denotes the set $[0, \infty)$. $\|\cdot\|$ denotes the Euclidean norm on $\mathbb{R}^{n} . \mathcal{R}[x]$ accounts for the set of polynomial functions with real coefficients in $x \in \mathbb{R}^{n}$, and $\Sigma \subset \mathcal{R}$ is the subset of polynomials with a sum of squares decomposition; i.e, $p \in \Sigma[x]$ if and only if there are $p_{i} \in \mathcal{R}[x], i \in\{1, \ldots, k\}$ such that $p=p_{i}^{2}+\cdots+p_{k}^{2}$. We denote by $\mathcal{C}^{m}(X)$, with $X \subseteq \mathbb{R}^{n}$, the space of $m$-times continuously differentiable functions and by $\partial^{m}=\frac{\partial^{m}}{\partial x^{m}}$ the derivatives up to order $m .2^{A}$ signifies the power set of $A$. Finally, for a finite set $A$, we denote by $\operatorname{co}\{A\}$ the convex hull of the set $A$.

\section{Preliminaries}

In this section, we discuss some preliminary mathematical notions and results that will be employed in the sequel.

\section{A. Whitney's Extension Problem}

Whitney's extension problem is concerned with the question of whether, given data on a function $f$, i.e., $\left\{\partial^{m} f_{i}\right\}_{i=1}^{N}$ corresponding to $\left\{x_{i}\right\}_{i=1}^{N}$ such that $\partial^{m} f_{i}=\partial^{m} f_{i}\left(x_{i}\right)$, one can find a $\mathcal{C}^{m}$-function that approximates $f$. It can be described as follows. Suppose we are given an arbitrary subset $D \subset \mathbb{R}^{n}$ and a function $f: D \rightarrow \mathbb{R}$. How can we determine whether there exists a function $F \in \mathcal{C}^{m}\left(\mathbb{R}^{n}\right)$ such that $F=f$ on $D$ ? Whitney indeed addressed this problem for the case $n=1$ [31]. Recently, [32] and [33] considered a more general problem. That is, given $\left\{f_{i}=f\left(x_{i}\right)\right\}_{i=1}^{N}$, the problem of computing a function $F \in \mathcal{C}^{m}\left(\mathbb{R}^{n}\right)$ and a real number $M \geq 0$ such that $\|F\|_{\mathcal{C}^{m}} \leq M \quad$ and $\quad|F(x)-f(x)| \leq M \sigma(x), \quad \forall x \in$ $D$. The function $\sigma: \mathbb{R}^{n} \rightarrow \mathbb{R}_{\geq 0}$ is determined by the problem under study and from "observations". The function also serves as a "tolerance". It implies that the graph of $F$ passes sufficiently close to the $N$ given data points.

Computing such a function $F$ in the general form is a cumbersome task and amounts to computing sets containing $F$ [34]. In this paper, instead of considering general interpolants of data, we focus on piecewise-polynomial approximations for which construction algorithms are widely available [35].

\section{B. Piecewise-Polynomial Approximation: B-Splines}

B-spline functions [36] have properties that make them very suitable candidates for function approximation. They can be efficiently computed in closed form based on available algorithms [35]. B-splines are widely employed in computer graphics, automated manufacturing, data fitting, computer graphics, and computer aided design [37].

A $p$ th degree B-spline function, $f(t)$, defined by $n$ control points (points that the curve passes through) and knots $\hat{t}_{i}$, $i=1,2, \ldots, n+p+1$, is given by

$$
f(t)=\sum_{i=1}^{n} \beta_{i} Q_{i, p}(t) .
$$

Knot vectors are sets of non-decreasing real numbers. The spacing between knots defines the shape of the curve along with the control points. Function $Q_{i, p}(t)$ is called $i$ th B-spline 
basis function of order $p$ and it can be described by the recursive equations

$$
Q_{i, 0}(t)= \begin{cases}1 & t \in\left[\hat{t}_{i}, \hat{t}_{i+1}\right) \\ 0 & \text { otherwise }\end{cases}
$$

and

$$
Q_{i, p}(t)=\frac{t-\hat{t}_{i}}{\hat{t}_{i+p}-\hat{t}_{i}} Q_{i, p-1}(t)+\frac{\hat{t}_{i+p+1}-t}{\hat{t}_{i+p+1}-\hat{t}_{i+1}} Q_{i+1, p-1}(t),
$$

defined using the Cox-de Boor algorithm [35]. First-order basis functions are evaluated using (2), followed by iterative evaluation of (3) until the desired order is reached. In contrast to Bézier curves, the number $n$ of control points of the curve, is independent of the order, $p$, providing more robustness for the generated paths topology.

Furthermore, the derivative of a B-spline $Q_{i, p}$ of degree $p$ is simply a function of $\mathrm{B}$-splines of degree $p-1$ :

$$
\frac{\mathrm{d} Q_{i, p}(t)}{\mathrm{d} t}=(p-1)\left(\frac{-Q_{i+1, p-1}}{t_{i+p}-t_{i+1}}+\frac{Q_{i, p-1}}{t_{i+p-1}-t_{i}}\right) .
$$

\section{Construction of Data-Driven Differential INCLUSIONS}

In this section, we bring forward a method that uses B-spline approximation of data and known regularity side information to construct a data-driven model. We call this model a datadriven differential inclusion. We show in the sequel that such data-driven differential inclusions enable safety analysis and controller synthesis.

\section{A. Piecewise-Polynomial Approximation of the Trajectories}

We study systems for which, at time instances $t_{1} \leq t_{2} \leq$ $\cdots \leq t_{N}$, samples of control and state values are available. In order to construct data-driven differential inclusions, we employ the tensor-product spline technique [38] which allows for efficient approximation of multi-variate systems. To this end, we parametrize the states as a function of $t$ and $u=\left(u_{1}, \ldots, u_{m}\right)^{T}, x(t, u)$. Furthermore, in order to obtain a control-affine model, we consider first-order spline (piecewise linear) functions of control inputs $u=\left(u_{1}, \ldots, u_{m}\right)^{T}$. That is, for each state, we have

$$
X_{l}(t)=\sum_{i=1}^{L} \sum_{j=1}^{m} c_{i, j}^{l} Q_{i, j}^{l}(t)\left(a_{0, j}^{l}+a_{1, j}^{l} u_{j}\right), \quad l=1,2, \ldots, n .
$$

We are interested in approximating the time evolution of the state. Computing the time-derivative of $X$ yields

$$
\begin{aligned}
& \dot{X}_{l}(t)=\sum_{i=1}^{L} \sum_{j=1}^{m} c_{i, j}^{l} \dot{Q}_{i, j}^{l}(t)\left(a_{0, j}^{l}+a_{1, j}^{l} u_{j}\right) \\
& =\sum_{i=1}^{L} \sum_{j=1}^{m} a_{0, j}^{l} c_{i, j}^{l} \dot{Q}_{i, j}^{l}(t)+\sum_{i=1}^{L} \sum_{j=1}^{m} a_{1, j}^{l} c_{i, j}^{l} \dot{Q}_{i, j}^{l}(t) u_{j},
\end{aligned}
$$

where $\dot{Q}_{i, j}^{l}(t)$ can be computed efficiently using the recursive formula (4). Define

$$
\begin{aligned}
f_{l}(t) & =\sum_{i=1}^{L} \sum_{j=1}^{m} a_{0, j}^{l} c_{i, j}^{l} \dot{Q}_{i, j}^{l}(t) \quad \text { and } \\
G_{l}(t) & =\left[\begin{array}{c}
\sum_{i=1}^{L} a_{1,1}^{l} c_{i, 1}^{l} \dot{Q}_{i, 1}^{l}(t) \\
\vdots \\
\sum_{i=1}^{L} a_{1, m}^{l} c_{i, m}^{l} \dot{Q}_{i, m}^{l}(t)
\end{array}\right] \in \mathbb{R}^{1 \times m} .
\end{aligned}
$$

Then, (6) can be rewritten as

$$
\dot{X}(t)=F(t)+G(t) u
$$

where $X=\left(X_{1}(t), \cdots, X_{n}(t)\right)^{T}, F=\left(f_{1}(t), \cdots, f_{n}(t)\right)^{T}$ and $G=\left(G_{1}(t), \cdots, G_{n}(t)\right)^{T}$.

\section{B. Data-Driven Differential Inclusions}

We are given samples of the state $\left\{x\left(t_{i}, u_{i}\right)\right\}_{i=1}^{N}$ at time instances $t_{1} \leq t_{2} \leq \cdots \leq t_{N}$. That is, information about only one trajectory is available. We consider state evolutions that belong to $\mathcal{C}^{2}\left(\mathbb{R}_{\geq 0}\right)$. Hence, $\dot{x}(t) \in \mathcal{C}^{1}\left(\mathbb{R}_{\geq 0}\right)$. We consider cases in which, in addition to state and input samples, some prior regularity knowledge (that we also call side information) on the state evolutions may be available. We capture such information by constraints in the form of

$$
\|x\|_{\mathcal{C}^{2}} \leq M,
$$

for a constant $M>0$. For mechanical systems, for example, the above constraint represents bounds on maximum acceleration.

In order to account for the uncertainty in approximating $\dot{x}$ with the function $\dot{X}$, we introduce a function $\sigma: \mathbb{R}_{\geq 0} \rightarrow \mathbb{R}_{\geq 0}$ such that

$$
|\dot{X}(t)-\dot{x}(t)| \leq M \sigma(t) .
$$

In the case of piecewise-polynomial approximation of the data, we have (7). From the side information (8), we have $\dot{X}_{-} \leq$ $\dot{x} \leq \dot{X}_{+}$, where $\dot{X}_{-}=\dot{X}(t)-M \sigma(t)$ and $\dot{X}_{+}=\dot{X}(t)+$ $M \bar{\sigma}(t)$ and $\dot{X}(t)$ is given as (7). Hence, the dynamics of the system for $t>t_{N}$, i.e., the convex cone generated by samples of the trajectory of the system and the side information, can be described by the following data-driven differential inclusion

$$
\left\{\begin{array}{l}
\dot{x}(t) \in \operatorname{co}\left\{\dot{X}_{-}(t), \dot{X}_{+}(t)\right\}, \\
x\left(t_{N}\right)=x_{N} .
\end{array}\right.
$$

Note that the above dynamics are dependent on the control signal through (7).

Differential inclusion (9) in fact over-approximates the dynamics after $t>t_{N}$. Note that the information on the system state is only available for $0<t<t_{N}$ and the regularity information ( $M$ and $\sigma$ ) provides the means using (9) to predict the behavior of system state. The rate of growth of the approximation error for $t>t_{N}$ can be approximated by the application of Gronwall's inequality [29]. This rate is a linear function of the Lipschitz constant of the interpolant. Nonetheless, cubic-spline polynomials are the best interpolants in terms of minimizing the Lipschitz constant for 


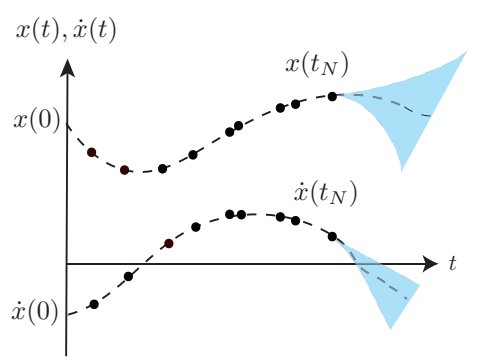

Fig. 1: Data samples (black dots), actual system state evolution (dashed lines), and the solution set of the data-driven differential inclusion (blue).

$\mathcal{C}^{2}$ trajectories [27]. Figure 1 illustrates the solution set of the differential inclusion (9) for a system with only one state and $\sigma(t)=1, \quad t>0$.

We are interested in solving the following safety analysis problem.

Problem 1: Consider the data-driven differential inclusion (9) with $u \equiv 0$. Given $\mathcal{X}_{u} \subset \mathbb{R}^{n}$ and $T>t_{N}$, check whether $x(T) \notin \mathcal{X}_{u}$.

In addition, we are interested in addressing the following safe controller synthesis problem.

Problem 2: Consider the data-driven differential inclusion (9). Given $\mathcal{X}_{u} \subset \mathbb{R}^{n}$ and $T>t_{N}$, find a feedback law $u(x)$ such that $x(T) \notin \mathcal{X}_{u}$.

In the next section, we discuss differential inclusions in the form of (9) and we address Problem 1 and Problem 2.

\section{SAFETy ANALYSiS AND SAFE CONTROLlER SYNTHESIS FOR DIFFERENTIAL INCLUSIONS}

We start by deriving a safety theorem based on barrier certificates for convex differential inclusions and then show how this result can be applied to the data-driven differential inclusion (9) for safe controller synthesis.

Let $\left\{f_{i}(t, x, u)\right\}_{i=1}^{m}$ be a family of (piecewise) smooth functions, where $f_{i}: \mathcal{T} \times \mathcal{X} \times \mathcal{U} \rightarrow \mathbb{R}^{n}$ with $\mathcal{X} \subseteq \mathbb{R}^{n}$, $\mathcal{T} \subseteq \mathbb{R}_{\geq 0}$, and $\mathcal{U} \subseteq \mathbb{R}^{m}$. We further assume $u \in \mathcal{L}^{\infty}$. Define $\mathcal{F}: \mathcal{T} \times \mathcal{X} \times \mathcal{U} \rightarrow 2^{\mathbb{R}^{n}}$ with

$$
\mathcal{F}(t, x, u)=c o\left\{f_{1}(t, x, u), \ldots, f_{m}(t, x, u)\right\} .
$$

Consider the following differential inclusion

$$
\left\{\begin{array}{l}
\dot{x} \in \mathcal{F}(t, x, u), \quad t \geq t_{0}, \\
x\left(t_{0}\right)=x_{0} .
\end{array}\right.
$$

Well-posedness conditions of differential inclusions [39, Theorem 1, p. 106] require the set-valued map $\mathcal{F}$ to be closed and convex for all $t, x$, and $u$, and also measurable in $u$. The set $\mathcal{F}(t, x, u)$ is closed and convex, because it is defined as the convex hull of a finite set. Furthermore, the mapping $\mathcal{F}(t, \cdot, u): \mathcal{X} \rightarrow 2^{\mathbb{R}^{n}}$ is upper hemi-continuous in $x$, for all $t$ and $u$, because it can be written as a convex combination of smooth mappings $f_{i}(t, \cdot, u)$. Finally, the mapping $\mathcal{F}(t, x, u)$ satisfies the one-sided Lipschitz condition

$$
\left(x_{1}-x_{2}\right)^{T}\left(v_{1}-v_{2}\right) \leq C\left\|x_{1}-x_{2}\right\|^{2}, \quad \forall t>0,
$$

for some $C>0$ and all $x_{1} \in \mathcal{X}, x_{2} \in \mathcal{X}, v_{1} \in \mathcal{F}\left(t, x_{1}, u\right)$ and $v_{2} \in \mathcal{F}\left(t, x_{2}, u\right)$, which follows from the fact that $\mathcal{F}(t, \cdot, u)$ is a convex hull of smooth and Lipschitz functions for all $t$ and $u$.

\section{A. Safety Analysis for Autonomous Differential Inclusions}

In order to propose a solution to Problem 1, we extend the concept of barrier certificates to differential inclusions.

Before stating the result, we require a definition of the derivative for set-valued maps [28]. Denote by

$$
D_{+} V(x)(f)=\liminf _{h \rightarrow 0^{+}, v \rightarrow f} \frac{V(x+h v)-V(x)}{h}
$$

the upper contingent derivative of $V$ at $x$ in the direction $f$. In particular, when $V$ is Gateaux differentiable and $\mathcal{F}=\{v\}$ is a singleton, $D_{+} V(x)$ coincides with the gradient

$$
D_{+} V(x)(v)=(\nabla V(x))^{T} v .
$$

Theorem 1. Consider differential inclusion (10) with $u \equiv 0$ and let $T>t_{0}$. If there exist a function $B \in \mathcal{C}^{1}\left(\mathbb{R}^{n} ; \mathbb{R}\right) \cap$ $\mathcal{C}^{1}\left(\mathbb{R}_{\geq 0} ; \mathbb{R}\right)$ and a positive definite function $W \in \mathcal{L}\left(\mathbb{R}^{n} \times\right.$ $\mathbb{R}_{\geq 0} ; \mathbb{R}_{\geq 0}$ ) such that

$$
\begin{gathered}
B(x(T), T)-B\left(x\left(t_{0}\right), t_{0}\right)>0, \quad x(T) \in \mathcal{X}_{u}, \\
D_{+} B(t, x)(v, 1) \leq-W(t, x), \quad t \in\left[t_{0}, T\right], \quad v \in \mathcal{F}(t, x),
\end{gathered}
$$

then the solutions of (10) satisfy $x(T) \notin \mathcal{X}_{u}$.

Proof. The proof is carried out by contradiction. Assume it holds that $x(T) \in \mathcal{X}_{u}$. Then, (11) implies that

$$
B(x(T), T)>B\left(x\left(t_{0}\right), t_{0}\right) .
$$

Furthermore, using the comparison theorem for differential inclusions [29, Proposition 8, p. 289] and inequality (12), we can infer that

$$
B(x(s), s)-B\left(x\left(t_{0}\right), t_{0}\right) \leq-\int_{t_{0}}^{s} W(x, \tau) d \tau \leq 0 .
$$

That is,

$$
B(x(s), s) \leq B\left(x\left(t_{N}\right), t_{N}\right) .
$$

Since $s$ was chosen arbitrary, this is a contradiction. Thus, the solutions of (10) satisfy $x(T) \notin \mathcal{X}_{u}$.

Theorem 1 presents conditions to check the safety of the solutions of a differential inclusion. The above formulation only necessitates the barrier certificate to have an upper contingent derivative. Hence, our formulation here includes non-smooth barrier certificates. The closest work on this class of barrier certificates is the recent research [40], wherein the authors considered $\mathrm{min} / \mathrm{max}$ barrier functions with application to control of multi-robot systems. Nonetheless, the formulation in [40] is based on fixing the barrier functions, i.e., the 0super level sets of the barrier functions define the unsafe sets. This assumption can be restrictive in many cases, in particular, 
general semi-algebraic sets. In this study, we do not make such assumptions on the structure of the barrier functions and we allow them to be variables in the computational formulation in Section V.

\section{B. Safe Controller Synthesis for Data-Driven Differential In- clusions}

Having established a safety analysis theorem for differential inclusions, we proceed by proposing conditions for safety analysis and safe controller synthesis for data-driven differential inclusion (9). We begin with the safety analysis result, which follows from Theorem 1. In other words, given the limited data over states up to some time $t_{N}$ and the regularity information, we verify whether the convex cone generated by that trajectory of the system (reconstructed from the data using cubic splines) and the side information behaves safely at a given time $T>t_{N}$.

Corollary 1. Consider differential inclusion (9) with $u \equiv$ 0 and let $T>t_{N}$. If there exist a function $B \in$ $\mathcal{C}^{1}\left(\mathbb{R}^{n} ; \mathbb{R}\right) \cap \mathcal{C}^{1}\left(\left[t_{N}, \infty\right) ; \mathbb{R}\right)$ and two positive definite functions $W_{+} \in \mathcal{L}\left(\mathbb{R}^{n} \times\left[t_{N}, \infty\right) ;\left[t_{N}, \infty\right)\right)$ and $W_{-} \in$ $\mathcal{L}\left(\mathbb{R}^{n} \times\left[t_{N}, \infty\right) ;\left[t_{N}, \infty\right)\right)$ such that

$$
\begin{array}{cc}
B(x(T), T)-B\left(x\left(t_{N}\right), t_{N}\right)>0, & x(T) \in \mathcal{X}_{u}, \\
D_{+} B(t, x)\left(\dot{X}_{-}, 1\right) \leq-W_{-}(t, x), & t \in\left[t_{N}, T\right], \\
D_{+} B(t, x)\left(\dot{X}_{+}, 1\right) \leq-W_{+}(t, x), & t \in\left[t_{N}, T\right],
\end{array}
$$

then the solutions of (9) satisfy $x(T) \notin \mathcal{X}_{u}$.

Proof. blueInequality (13) ensures that (11) holds. Multiplying both sides of inequality (14) with a constant $0 \leq \alpha_{-} \leq 1$ and inequality (15) with a constant $0 \leq \alpha_{+} \leq 1$ such that $\alpha_{-}+$ $\alpha_{+}=1$ and adding them, we obtain $\alpha_{-} D_{+} B(t, x)\left(\dot{X}_{-}, 1\right)+$ $\alpha_{+} D_{+} B(t, x)\left(\dot{X}_{+}, 1\right) \leq-\alpha_{-} W_{-}(t, x)-\alpha_{+} W_{+}(t, x)$. Since $D_{+}$is a linear operator, we have $D_{+} B(t, x)\left(\alpha_{-} \dot{X}_{-}+\right.$ $\left.\alpha_{+} \dot{X}_{+}, 1\right) \leq D_{+} B(t, x)\left(\alpha_{-} \dot{X}_{-}\right)+D_{+} B(t, x)\left(\alpha_{-} \dot{X}_{+}\right) \leq$ $-\alpha_{-} W_{-}(t, x)-\alpha_{+} W_{+}(t, x)$, where, in the last line above, we applied inequalities (14) and (15). Let $W(t, x)=$ $\min \left\{W_{-}(t, x), W_{+}(t, x)\right\}$. We obtain $D_{+} B(t, x)\left(\alpha_{-} \dot{X}_{-}+\right.$ $\left.\alpha_{+} \dot{X}_{+}, 1\right) \leq-\alpha_{-} W_{-}(t, x)-\alpha_{+} W_{+}(t, x) \leq-\left(\alpha_{-}+\right.$ $\left.\alpha_{+}\right) W(t, x)=-W(t, x)$. That is, $D_{+} B(t, x)(v, 1) \leq$ $-W(t, x), \quad v \in \operatorname{co}\left\{\dot{X}_{-}, \dot{X}_{+}\right\}$. Thus inequality (12) is also satisfied. This completes the proof.

We show in Section $\mathrm{V}$ that if we parametrize $B$ and $W$ by polynomials of fixed degree and let $\mathcal{X}_{u}$ be a semi-algebraic set, then we can check inequalities (13) through (15) using sum of squares programming.

We next address Problem 2 by proposing a method to design controllers such that solutions to (7) are safe with respect to a given unsafe set $\mathcal{X}_{u}$ for all $t>t_{N}$. In other words, we address Problem 2.

Corollary 2. Consider differential inclusion (9) and let $T>t_{N}$. If there exist a function
$B \in \mathcal{C}^{1}\left(\mathbb{R}^{n} ; \mathbb{R}\right) \cap \mathcal{C}^{1}\left(\left[t_{N}, \infty\right) ; \mathbb{R}\right)$ and a positive definite function $W \in \mathcal{L}\left(\mathbb{R}^{n} \times\left[t_{N}, \infty\right) ;\left[t_{N}, \infty\right)\right)$ such that

$$
B(x(T), T)-B\left(x\left(t_{N}\right), t_{N}\right)>0, \quad x(T) \in \mathcal{X}_{u},
$$

$$
\begin{aligned}
\frac{\partial B}{\partial t}+\left(\frac{\partial B}{\partial x}\right)^{T}(f-M \sigma) \leq & -W(t, x) \\
& \forall x \in \mathcal{X}, \quad \forall t \in\left[t_{N}, T\right]
\end{aligned}
$$

$$
\begin{aligned}
\frac{\partial B}{\partial t}+\left(\frac{\partial B}{\partial x}\right)^{T}(f+M \sigma) \leq & -W(t, x) \\
& \forall x \in \mathcal{X}, \quad \forall t \in\left[t_{N}, T\right]
\end{aligned}
$$

and

$$
\left(\frac{\partial B}{\partial x}\right)^{T} g \neq 0, \quad \forall x \in \mathcal{X}, \quad \forall t \in\left[t_{N}, T\right]
$$

then the controller

$$
u=g^{T} \frac{\partial B}{\partial x}\left(\left(\frac{\partial B}{\partial x}\right)^{T} g g^{T} \frac{\partial B}{\partial x}\right)^{-1} W(t, x),
$$

renders the solutions of (9) safe, i.e., $x(t) \notin \mathcal{X}_{u}$ for all $t \in$ $\left[t_{N}, T\right]$.

Proof. The proof follows by applying Corollary 2 to system (7) with $W_{-}=W_{+}=W$. Inequality (16) ensures that (13) holds. Computing the directional derivative of $B$ along the solutions of system (7) and using the linearity property of the $D_{+}$operator we have

$$
\begin{gathered}
D_{+} B(t, x)(f(t)+g(t) u \pm M \sigma(t), 1) \\
=\frac{\partial B}{\partial t}+\left(\frac{\partial B}{\partial x}\right)^{T}(f+g u \pm M \sigma) \\
=\frac{\partial B}{\partial t}+\left(\frac{\partial B}{\partial x}\right)^{T}(f \pm M \sigma)+\left(\frac{\partial B}{\partial x}\right)^{T} g u
\end{gathered}
$$

Note that if $\left(\frac{\partial B}{\partial x}\right)^{T} g=0$, we cannot design $u$ such that makes the last line of (21) negative definite, i.e., to ensure safety. Noting that (19) holds and substituting the controller (20) in the last line of (21), we obtain

$$
\begin{array}{rl}
\frac{\partial B}{\partial t}+\left(\frac{\partial B}{\partial x}\right)^{T} & (f \pm M \sigma) \\
+\left(\frac{\partial B}{\partial x}\right)^{T} & g g^{T} \frac{\partial B}{\partial x}\left(\left(\frac{\partial B}{\partial x}\right)^{T} g g^{T} \frac{\partial B}{\partial x}\right)^{-1} W(t, x) \\
& =\frac{\partial B}{\partial t}+\left(\frac{\partial B}{\partial x}\right)^{T}(f \pm M \sigma) \\
& +W(t, x)-W(t, x)+W(t, x) \leq 0
\end{array}
$$

where, in the last line inequality above, we used the fact that (17) and (18) hold. Thus, $D_{+} B(t, x)(f(t)+g(t) \pm$ $M \sigma(t), 1) \leq 0$. 
Let $0 \leq \alpha_{1}, \alpha_{2} \leq 1$ satisfying $\alpha_{1}+\alpha_{2}=1$. Then,

$$
\begin{aligned}
& \alpha_{1} D_{+} B(t, x)(f(t)+g(t)+M \sigma(t), 1) \\
& +\alpha_{2} D_{+} B(t, x)(f(t)+g(t)-M \sigma(t), 1) \\
& =D_{+} B(t, x)\left(\alpha_{1}(f(t)+g(t)+M \sigma(t)), 1\right) \\
& +D_{+} B(t, x)\left(\alpha_{2}(f(t)+g(t)-M \sigma(t)), 1\right) \\
& =D_{+} B(t, x)\left(\alpha_{1}(f(t)+g(t)+M \sigma(t))\right. \\
& \left.+\alpha_{2}(f(t)+g(t)-M \sigma(t)), 1\right) \leq 0,
\end{aligned}
$$

where in the last line we used (22).

The goal of this controller is to force the solutions of the data-driven differential inclusion (9) to avoid a prespecified unsafe set $\mathcal{X}_{u}$ at a particular point in time, for a time period, or for all time after its activation. If such a controller exists a given horizon $T$, then from Corollary 2 we infer that $x(t) \notin \mathcal{X}_{u}$ for all $t \in\left[t_{N}, T\right]$. Note that the unsafe set $\mathcal{X}_{u}$ can defined such that the solutions of the data-driven differential inclusion (9) avoid blow up over a time span.

\section{Computational Method}

In this section, we propose computational methods to address the analysis and the synthesis problems. Piecewise polynomial interpolation leads to a computational formulation based on polynomial optimization or sum-of-squares programs.

Assuming $\sigma \in \Sigma[t]$, (9) becomes a differential inclusion with polynomial vector fields. The next lemma, which is based on the application of Putinar's Positivestellensatz [41], [42], presents conditions in terms of polynomial positivity that can be efficiently checked via semi-definite programs (SDPs) [43].

Lemma 1. Consider the differential inclusion (9) with $u \equiv 0$ and the following semi-algebraic unsafe set

$$
\mathcal{X}_{u}=\left\{x \mid l_{i}(x) \leq 0, i=1,2, \ldots, n_{c}\right\},
$$

where $l_{i} \in \mathcal{R}[x]$. If there exist functions $B \in \mathcal{R}[x, t], W_{-} \in$ $\Sigma[x, t], W_{+} \in \Sigma[x, t], m_{i} \in \Sigma[x, t], i=1,2, s_{i} \in \Sigma[x, t]$, $i=1, \ldots, n_{c}$ and a positive constant $c>0$, such that

$$
\begin{aligned}
B(x(T), T) & -B\left(x\left(t_{N}\right), t_{N}\right) \\
& +\sum_{i=1}^{n_{c}} s_{i}(x(T)) l_{i}(x(T))-c \in \Sigma[x(T)]
\end{aligned}
$$

and

$$
\begin{aligned}
& -\frac{\partial B}{\partial t}-\left(\frac{\partial B}{\partial x}\right)^{T} \dot{X}_{-}-W_{-}(t, x) \\
& -m_{1}(t, x)\left(t-t_{N}\right)(t-T) \in \Sigma[x, t], \\
& -\frac{\partial B}{\partial t}-\left(\frac{\partial B}{\partial x}\right)^{T} \dot{X}_{+}-W_{+}(t, x) \\
& -m_{2}(t, x)\left(t-t_{N}\right)(t-T) \in \Sigma[x, t],
\end{aligned}
$$

then the solutions to (9) satisfy $x(T) \notin \mathcal{X}_{u}$.
Proof. Applying Putinar's Positivstellensatz, condition (24) implies that $B(x(T), T)-B\left(x\left(t_{N}\right), t_{N}\right)>0$, for all $x(T) \in$ $\mathcal{X}_{u}$ as in (13). Thus, inequality (13) holds. Moreover, since $B \in \mathcal{R}[x, t]$ and thus smooth, we have $D_{+} B(t, x)\left(\dot{X}_{-}, 1\right)=$ $\frac{\partial B}{\partial t}+\left(\frac{\partial B}{\partial x}\right)^{T} \dot{X}_{-}$. Hence, from condition (25), we have $D_{+} B(t, x)\left(\dot{X}_{-}, 1\right) \leq-W_{-}(t, x), \quad t \in\left[t_{N}, T\right]$. Therefore, inequality (14) is satisfied. In a similar manner, we can show that (15) holds as well. Analogously, we can show that (26) implies that (15) is satisfied. Then, from Corollary 2, the solutions to (9) satisfy $x(T) \notin \mathcal{X}_{u}$.

We now focus on formulating sum of squares conditions to synthesize a safe controller as highlighted in Corollary 2 . The following result can be proved by a direct application of Positivstellensatz.

Lemma 2. Consider the differential inclusion (9) and the following semi-algebraic unsafe set

$$
\mathcal{X}_{u}=\left\{x \mid l_{i}(x) \leq 0, i=1,2, \ldots, n_{c}\right\},
$$

where $l_{i} \in \mathcal{R}[x]$. If there exist functions $B \in \mathcal{R}[x, t], W \in$ $\Sigma[x, t], m_{i} \in \Sigma[x, t], i=1,2, s_{i} \in \Sigma[x, t], i=1, \ldots, n_{c}$, $q_{1} \in \mathcal{R}[x, t], q_{2} \in \Sigma[x, t]$ and a positive constant $c>0$ such that

$$
\begin{aligned}
& B(x(T), T)-B\left(x\left(t_{N}\right), t_{N}\right) \\
& \quad+\sum_{i=1}^{n_{c}} s_{i}(x(T)) l_{i}(x(T))-c \in \Sigma[x(T)], \\
& -\frac{\partial B}{\partial t}-\left(\frac{\partial B}{\partial x}\right)^{T}(f-M \sigma) \\
& \quad-W(t, x)-m_{1}(t, x)\left(t-t_{N}\right)(t-T) \in \Sigma[x, t], \\
& -\frac{\partial B}{\partial t}-\left(\frac{\partial B}{\partial x}\right)^{T}(f+M \sigma) \\
& \quad-W(t, x)-m_{2}(t, x)\left(t-t_{N}\right)(t-T) \in \Sigma[x, t],
\end{aligned}
$$

and

$$
-q_{1}\left(\left(\frac{\partial B}{\partial x}\right)^{T} g\right)-q_{2} \in \Sigma[x, t],
$$

then the solutions to (9) satisfy $x(T) \notin \mathcal{X}_{u}$.

Note that sum of squares constraint (31) is bilinear in variables $B$ and $q_{1}$. In practice, we fix the polynomial $q_{1}$ and look for a barrier certificate such that (28) to (31) are satisfied. In many cases such as the examples given in Section VI, this constraint holds for a computed barrier certificate $B$.

\section{NUMERICAL RESULTS}

In this section, we illustrate the proposed method using an example of a safe-landing scenario for an aircraft with critical failure. In the following example, we used the parser SOSTOOLs [44] to cast the polynomial inequalities into semidefinite programs and then we used Sedumi [45] to solve the resultant SDPs.

We consider a point mass longitudinal model of an aircraft subject to the gravity force $m g$ with $m$ being the mass and 


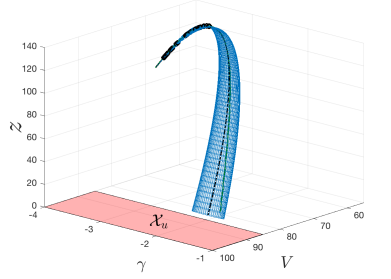

Fig. 2: The data points $\left\{x\left(t_{i}\right)\right\}_{i=1}^{25}$ (black circles), the piecewise-polynomial approximation of the state $X(t)$ (black line), the solution set of the data-driven differential inclusion (the meshed cone) and the actual solution of the system (green).

$g=9.8 \mathrm{~ms}^{-2}$, thrust $\tau$, lift $L$, and drag $D$. The equations of motion are then described by

$$
\begin{aligned}
\dot{V} & =\frac{1}{m}[\tau \cos (\alpha)-D(\alpha, V)-m g \sin (\gamma)], \\
\dot{\gamma} & =\frac{1}{m V}[\tau \sin (\alpha)+L(\alpha, V)-m g \cos (\gamma)], \\
\dot{z} & =V \sin (\gamma),
\end{aligned}
$$

where $V$ the velocity, $\gamma$ the flight path angle and $z$ the altitude are the states of the system. The angle of attack $\alpha$ and thrust $\tau$ are the inputs of the system.

Typically, landing is operated at $\tau_{\text {idle }}=0.2 \tau_{\max }$ where $\tau_{\max }$ is the maximal thrust. This value enables the aircraft to counteract the drag due to flaps, slats and landing gear. Most of the parameters for the DC9-30 can be found in the literature. The values of the numerical parameters used for the DC9-30 in this flight configuration are $m=60,000(\mathrm{~kg})$, and $\tau_{\max }=160,000(N)$. The lift and drag forces are given as $L(\alpha, V)=68.6(1.25+4.2 \alpha) V^{2}$ and $D(\alpha, V)=$ $\left[2.7+3.08(1.25+4.2 \alpha)^{2}\right] V^{2}$, respectively (both in Newtons).

For safety analysis and controller synthesis, we consider two separate aircraft failure scenarios in which the aircraft model (32) is not valid anymore and we use data-driven differential inclusion models instead. In both cases, we are interested in the safe landing speed of the aircraft $V_{\text {safe }}$ as it reaches the ground $z=0$. Therefore, we define the unsafe set as $\mathrm{X}_{u}=\left\{(V, \gamma, z) \mid V>V_{\text {safe }}, z=0\right\}$. We consider the following regularity side information $\|x\|_{\mathcal{C}^{1}} \leq$ $10, \quad|\dot{X}(t)-\dot{x}(t)| \leq 10$.

In the first scenario, we model wing failure by sudden drop in the lift force $L$ to $0.2 L$ at $t=0.5(s)$. We collect 25 data samples until $t=2(s)$ and we are interested in checking whether the aircraft satisfies the safe landing speed at the time of landing. Using Lemma 1, we found a barrier certificate of degree 5 proving that the system is safe. Figure 2 illustrates the collected data, the actual system state evolution and the solutions of the data-driven differential inclusion given the side information.

In the second scenario, we consider an engine control failure which is modeled as a sudden surge in thrust (to $0.8 \tau_{\max }$ from $0.2 \tau_{\max }$ ) at $t=0.5(\mathrm{~s}) .25$ data samples are collected non-uniformly until $t=2(s)$. The simulation results show

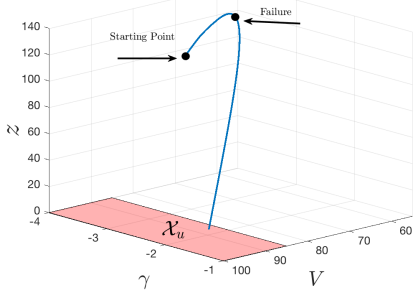

Fig. 3: The solution of the plane system (blue) and the unsafe set (red surface).

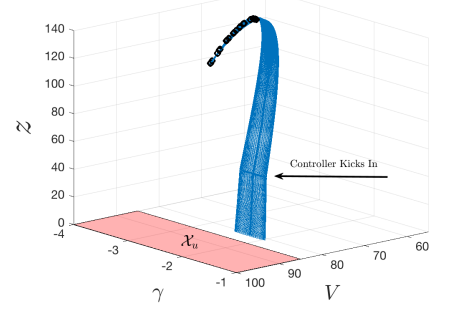

Fig. 4: The solution of the data driven differential inclusion system (meshed surface), data points (black circles) and the unsafe set (red surface). Note that, as the safe controller kicks in, the state evolution of the system becomes safe.

that the system trajectories without the controller are not safe as shown on Figure 3. A data-driven differential inclusion is constructed using the side information and piecewise polynomial interpolation. We then allow $T_{g}=5(s)$ to calculate the safe controller using Lemma 2. Figure 4 shows the result of applying the safe controller obtained based on Lemma 2 with certificates $B$ and $W$ of degree 3 . Hence, the safe controller is able to ensure safe landing despite the critical failure of the aircraft.

In order to understand the effect of picking different numbers of data samples with the same sampling period, and different sampling periods with the same number of data points, the interested reader is referred to the follow up work [46].

\section{CONCLUSIONS}

\section{A. Conclusions}

blue We considered the problem of safety analysis and controller synthesis for safety of systems for which only limited data and some regularity side information on system states are available. We reformulated the problem into safety analysis and safe controller synthesis of differential inclusions. We proposed a solution established upon an extension of barrier certificates for differential inclusions.

\section{B. Future Work}

If some knowledge on the physics of the underlying system is known a priori, learning can be done more efficiently. In [46], we demonstrated, if we know that the system follows Lagrangian or Hamiltonian mechanics, learning can be done using quadratic programming. 
blueFor certain classes of dynamical systems predicting the behavior based on segments of the trajectory is more conservative (for example, see the predictions of the chaotic solutions of a Van der Pol Oscillator in [30]). However, even for chaotic systems, if one can show that a barrier certificate exist for the conservative differential inclusion, we can still verify safety. Indeed, we demonstrated that the performance of the predictions based on the data driven differential inclusions is a function of the Lipschitz continuity of the solutions [46, Appendix B].

In this study, we assumed the measurements of the states are not noisy. In many practical situations, this is not the case and sensor measurements are subject to measurement noise, say due to heat. In this setting, safety analysis requires side information in the probabilistic sense. In this respect, one can use notions such as spline smoothing [47].

\section{REFERENCES}

[1] F. L. Lewis, D. Vrabie, and K. G. Vamvoudakis, "Reinforcement learning and feedback control: Using natural decision methods to design optimal adaptive controllers," IEEE Control Systems, vol. 32, no. 6, pp. 76-105, 2012

[2] I. Lenz and A. Saxena, "Deepmpc: Learning deep latent features for model predictive control," in In Robotics Systems and Science, 2015.

[3] D. Leone, "How an israeli F-15 eagle managed to land with one wing," 2014. [Online]. Available: https://theaviationist.com/2014/09/15/f-15lands-with-one-wing/

[4] M. Schmidt and H. Lipson, "Distilling free-form natural laws from experimental data," Science, no. 5923, pp. 81-85, 2009.

[5] D. Hills, A. Grutter, and J. Hudson, "An algorithm for discovering Lagrangians automatically from data," Peer J. Computer Science, no. $1: \mathrm{e} 31,2015$

[6] G. Tran and R. Ward, "Exact recovery of chaotic systems from highly corrupted data," arXiv preprint arXiv:1607.01067, 2016.

[7] L. Ljung, "Perspectives on system identification," Annual Reviews in Control, vol. 34, no. 1, pp. $1-12,2010$.

[8] S. Aghli and C. Heckman, "Online system identification and calibration of dynamic models for autonomous ground vehicles," in 2018 IEEE International Conference on Robotics and Automation (ICRA). IEEE, 2018, pp. 4933-4939.

[9] G. C. Karras, P. Marantos, C. P. Bechlioulis, and K. J. Kyriakopoulos, "Unsupervised online system identification for underwater robotic vehicles," IEEE Journal of Oceanic Engineering, 2018.

[10] G. Tao, "Multivariable adaptive control: A survey," Automatica, vol. 50, no. 11 , pp. $2737-2764,2014$

[11] H. Kim and A. Ng, "Stable adaptive control with online learning," in Advances in Neural Information Processing Systems, 2005, pp. 977-984.

[12] B. Kramer, B. Peherstorfer, and K. Willcox, "Feedback control for systems with uncertain parameters using online-adaptive reduced models," SIAM Journal on Applied Dynamical Systems, vol. 16, no. 3, pp. 1563 1586, 2017.

[13] C. J. Tomlin, I. Mitchell, A. M. Bayen, and M. Oishi, "Computational techniques for the verification of hybrid systems," Proceedings of the IEEE, vol. 91, no. 7, pp. 986-1001, July 2003.

[14] S. Prajna, "Barrier certificates for nonlinear model validation," Automatica, vol. 42, no. 1, pp. $117-126,2006$.

[15] M. Ahmadi, A. W. K. Harris, and A. Papachristodoulou, "An optimization-based method for bounding state functionals of nonlinear stochastic systems," in Decision and Control (CDC), 2016 IEEE 55th Conference on. IEEE, 2016, pp. 5342-5347.

[16] M. Ahmadi, G. Valmorbida, and A. Papachristodoulou, "Safety verification for distributed parameter systems using barrier functionals," Systems \& Control Letters, vol. 108, no. Supplement C, pp. 33 - 39, 2017.

[17] R. L. Christensen, R. Wisniewski, and K. K. Sorensen, "Safety verification of refrigeration containers using barrier certificates," in 2016 IEEE Conference on Computer Aided Control System Design (CACSD), Sept 2016, pp. 635-640.

[18] R. Wisniewski and C. Sloth, "Converse barrier certificate theorems," IEEE Transactions on Automatic Control, vol. 61, no. 5, pp. 1356-1361, May 2016.
[19] S. Han, U. Topcu, and G. J. Pappas, "A sublinear algorithm for barriercertificate-based data-driven model validation of dynamical systems," in 2015 54th IEEE Conference on Decision and Control (CDC), Dec 2015, pp. 2049-2054.

[20] P. Wieland and F. Allgower, "Constructive safety using control barrier functions," IFAC Proceedings Volumes, vol. 40, no. 12, pp. 462 - 467, 2007, 7th IFAC Symposium on Nonlinear Control Systems.

[21] E. D. Sontag, "A universal construction of Arstein's theorem on nonlinear stabilization," Systems \& Control Letters, pp. 117-123, 1989.

[22] M. Z. Romdlony and B. Jayawardhana, "Stabilization with guaranteed safety using control Lyapunov-barrier function," Automatica, vol. 66 , no. Supplement C, pp. $39-47,2016$.

[23] A. D. Ames, X. Xu, J. W. Grizzle, and P. Tabuada, "Control barrier function based quadratic programs for safety critical systems," IEEE Transactions on Automatic Control, 2016.

[24] Q. Nguyen and K. Sreenath, "Optimal robust control for constrained nonlinear hybrid systems with application to bipedal locomotion," in 2016 American Control Conference (ACC). IEEE, 2016, pp. 48074813.

[25] R. Cheng, G. Orosz, R. M. Murray, and J. W. Burdick, "End-to-end safe reinforcement learning through barrier functions for safety-critical continuous control tasks," arXiv preprint arXiv:1903.08792, 2019.

[26] C. Fefferman, Smooth Interpolation of Data by Efficient Algorithms. Boston: Birkhäuser Boston, 2013, pp. 71-84.

[27] A. Herbert-Voss, M. J. Hirn, and F. McCollum, "Computing minimal interpolants in $C^{1,1}\left(\mathbb{R}^{d}\right)$," Revista Matematica Iberoamericana, vol. 33, no. 1, pp. 22-69, 2017.

[28] J. P. Aubin and H. Frankowska, Set-Valued Analysis. Boston: Birkhäuser, 2009.

[29] J. P. Aubin and A. Celina, Differential Inclusions. Springer-Verlag, Berlin, 1984.

[30] M. Ahmadi, A. Israel, and U. Topcu, "Safety-assessment for physicallyviable data-driven models," in The 56th IEEE Conference on Decision and Control, Melbourne, Australia, Dec 2017.

[31] H. Whitney, "Analytic extensions of differentiable functions defined in closed sets," Transactions of the American Mathematical Society, vol. 36, no. 1, pp. 63-89, 1934.

[32] C. L. Fefferman, "A sharp form of Whitney's extension theorem," Annals of Mathematics, vol. 161, no. 1, pp. 509-577, 2005.

[33] C. Fefferman and B. Klartag, "Fitting a $C^{m}$-smooth function to data i," Rev. Mat. Iberoamericana, vol. 25, no. 1, pp. 49-273, 2009.

[34] C. Fefferman and A. Israel, "The jet of an interpolant on a finite set," Rev. Mat. Iberoamericana, vol. 27, no. 1, pp. 355-360, 2011.

[35] C. de Boor, A Practical Guide to Splines, ser. Applied Mathematical Sciences. Springer New York, 2001.

[36] P. Lancaster and K. Šalkauskas, Curve and Surface Fitting: An Introduction, ser. Computational mathematics and applications. Academic Press, 1986.

[37] G. Farin, "Cubic spline interpolation," in Curves and Surfaces for Computer-Aided Geometric Design (Third Edition), third edition ed., G. Farin, Ed. Boston: Academic Press, 1993, pp. 133 - 155.

[38] E. Grosse, "Tensor spline approximation," Linear Algebra and its Applications, vol. 34, no. Supplement C, pp. 29 - 41, 1980.

[39] A. F. Filippov, Differential Equations with Discontinuous Right-Hand Sides, ser. Mathematics and Its Applications. Kluwer, 1988.

[40] P. Glotfelter, J. Cortes, and M. Egerstedt, "Nonsmooth barrier functions with applications to multi-robot systems," IEEE Control Systems Letters, vol. 1, no. 2, pp. 310-315, Oct 2017.

[41] M. Putinar, "Positive polynomials on compact semi-algebraic sets," Indiana University Mathematics Journal, vol. 42, no. 3, pp. 969-984, 1993.

[42] M. Schweighofer, "Optimization of polynomials on compact semialgebraic sets," SIAM Journal on Optimization, vol. 15, no. 3, pp. 805-825, 2005.

[43] P. Parrilo, "Structured semidefinite programs and semialgebraic geometry methods in robustness and optimization," Ph.D. dissertation, California Institute of Technology, 2000.

[44] S. Prajna, A. Papachristodoulou, P. Seiler, and P. Parrilo, "SOSTOOLS: Sum of squares optimization toolbox for MATLAB V3.00," 2013.

[45] J. F. Sturm, "Using sedumi 1.02, a matlab toolbox for optimization over symmetric cones," 1998.

[46] M. Ahmadi, C. Rowley, and U. Topcu, "Control-Oriented Learning of Lagrangian and Hamiltonian Systems," in American Control Conference, 2018.

[47] R. L. Smith, L. M. Howser, and J. M. Price, A smoothing algorithm using cubic spline functions. Washington: National Aeronautics and Space Administration, Technical Report, 1974. 